\section{Optical Coherence Tomography Findings Facilitate the Diagnosis of Autosomal Recessive Spastic Ataxia of Charlevoix-Saguenay}

\section{Dear Editor,}

In patients with slowly progressing ataxia, molecular genetic investigation can be considered for the identification of underlying cause. Before considering genetic tests, comprehensive neurological and ophthalmological investigations should be done first. In this report, we present a rare case of a patient who was diagnosed with autosomal recessive spastic ataxia of Charlevoix-Saguenay (ARSACS) based on thickened retinal nerve fiber layers (RNFLs) and whole genome sequencing.

A 53-year-old male was referred from the department of neurology for diplopia of 6 months' duration. His past medical history was significant for the gait disturbance since age of 10. The patient had experienced progressive gait difficulty with frequent falls and was unable to walk independently at the age of 17 . At the age of 40 years, dysarthria and dysphagia developed, and brain magnetic resonance imaging (MRI) showed cerebellar atrophy. His younger sister had similar symptoms, which indicated an autosomal recessive trait. Best-corrected visual acuity was $20 / 25$ in the right eye and $20 / 40$ in the left eye. Slit lamp examination revealed pseudophakia in the right eye and cortical opacity in the left eye. The patient had a full range of eye movement, but defective pursuit movements were noted. Extraocular motility examination showed 25 prism diopters exotropia at near with Krimsky methods. Dilated fundus examination showed diffuse peripapillary retinal nerve fiber striations in both eyes. Optical coherence tomography (OCT) revealed thickening of RNFLs in both eyes (Fig. 1A-1D). Neurophysiologic studies were consistent with predominantly demyelinating sensori-motor

Received: March 19, 2021 Final revision: May 17, 2021

Accepted: May 21, 2021 polyneuropathy. Brain MRI showed cerebellar atrophy and pontine linear hypointense striations (Fig. 1). Genetic tests for Friedreich's ataxia (FRDA) and for the most common repeat expansion (spinocerebellar ataxia [SCA] 1, 2, 3, 6, 7, 8,17 , and dentatorubro-pallidoluysian atrophy) were all negative. Alpha-fetoprotein and vitamin E level $(7.4 \mathrm{mg} / \mathrm{L}$, normal 5.5-17.0) were normal, and echocardiography was also normal. Whole genome sequencing was performed, and it revealed two compound heterozygous variants c.7272C>A:p.(Cys2424*), c.11319_11321del:p.(Arg3774del) in the SACS gene (NM_014363.4). These two variants were absent in gnom AD and predicted to be deleterious (CADD score 33 and 19.39, respectively). The former variant was previously reported [1], and it is considered as pathogenic. The latter in-frame deletion is a deletion of highly conserved arginine, deposited in ClinVar (VCV000853474) and considered as likely pathogenic (PM1, PM2, PM4, PP3, and PP4). Due to absence of his parents, we could not determine the phase of two variants.

ARSACS was first described in the families of the Charlevoix and Saguenay regions of Quebec, Canada [2]. Unsteady walking is the first symptom of ARSACS, followed by slowly progressive muscle spasticity, problems with coordination and reduced sensation and weakness in the extremities [2]. Affected individuals also had slurred speech, saccadic pursuit, and nystagmus. Although the typical age of onset is early childhood $(3.4 \pm 1.6$ years), wide variability of age at onset (infancy to $>40$ years) has also been described [3]. The causative gene was found to be a mutation in the $S A C S$ gene, which encodes for the sacsin. Its function is thought to be essential for neurofilament arrangement and mitochondrial dynamics. Previous literature reported that only one patient had diplopia among 17 patients with ARSACS [4]. The cerebellar atrophy may be responsible for the diplopia. The average peripapillary RNFL thickness of $119 \mu \mathrm{m}$ showed the greatest differentiation between ARSACS and other causes [4].

Given the diagnostic complexity in hereditary ataxia, accurate phenotyping is essential to make a correct diagnosis. Ataxia-telangiectasia, FRDA, and SCA are differential diagnoses to be considered. The diagnosis of ataxia-telangiectasia was deferred because conjunctival telangiectasia was absent in this patient. OCT findings revealed RNFL thinning 

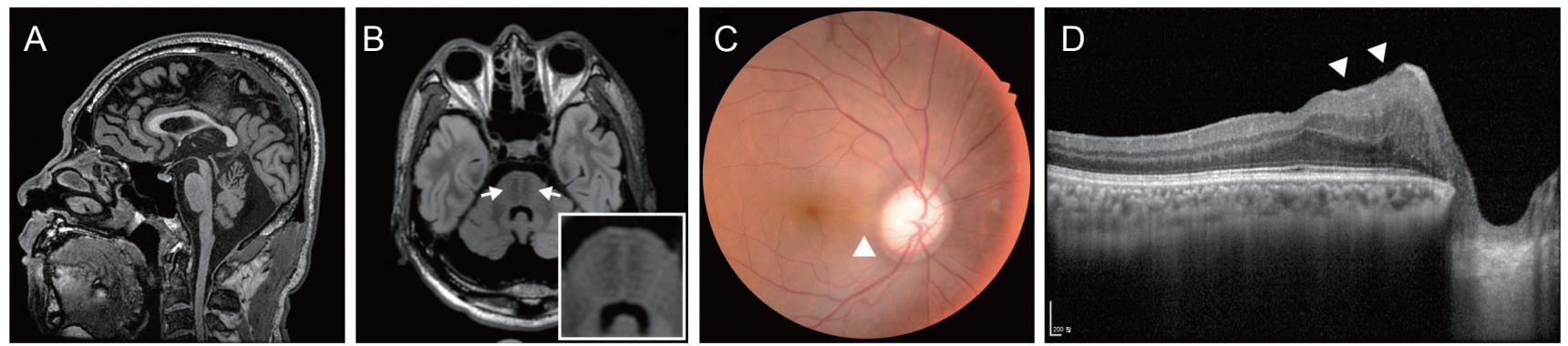

Fig. 1. Autosomal recessive spastic ataxia of Charlevoix-Saguenay. (A) Sagittal T1 image shows cerebellar vermis atrophy. (B) Axial T1 sequence shows bilateral pontine linear hypointense lesion (arrows). (C) Fundus photograph reveals retinal striations (arrowhead) nasal to the fovea (right eye was shown) (D) Optical coherence tomography showing elevated fovea pit and thickened retinal nerve fiber layer (arrowheads) nasal to the fovea, corresponding to the retinal striations. Informed consent for publication of the clinical images was obtained from the patient.

in FRDA [5]. In addition, substantial portion of patients with FRDA developed cardiomyopathy, which is inconsistent with our case [5]. The SCA 7 typically presented with cone dystrophy with ataxia, and this disorder is inherited as dominant trait. If thickening of RNFL is observed in patients with progressive ataxia, ARSACS could be the cause.

Although ARSACS has no specific treatment, prompt and appropriate diagnosis bears important prognostic implications. In patients with hereditary ataxia, pontine linear hypointensities on MRI and RNFL thickening on OCT may provide important clues for diagnosis. Comprehensive ophthalmological assessment in conjunction with pertinent medical history may guide to differentiate the cause of hereditary ataxia, and this makes us to avoid unnecessary trinucleotide repeat expansion tests for SCAs.

\section{Hyuna Cho}

Institute of Vision Research, Department of Ophthalmology, Gangnam Severance Hospital, Yonsei University College of Medicine, Seoul, Korea

\section{Chul Hyoung Lyoo}

Department of Neurology, Gangnam Severance Hospital, Yonsei University College of Medicine, Seoul, Korea

\section{Sung Eun Park, Yuri Seo, Sueng-Han Han} Institute of Vision Research, Department of Ophthalmology, Severance Hospital, Yonsei University College of Medicine, Seoul, Korea

\section{Jinu Han}

Institute of Vision Research, Department of Ophthalmology, Gangnam Severance Hospital, Yonsei University College of Medicine, Seoul, Korea

E-mail: jinuhan@yuhs.ac

\section{Conflict of Interest}

No potential conflict of interest relevant to this article was reported.

\section{Acknowledgements}

This study was supported by the Korea Disease Control and Prevention Agency (2018-ER6902-02 and 2019-NG051-01) and National Research Foundation of Korea (NRF) grant funded by the Korea government (MSIT) (2020R1C1C1007965).

\section{References}

1. Thiffault I, Dicaire MJ, Tetreault M, et al. Diversity of ARSACS mutations in French-Canadians. Can J Neurol Sci 2013;40:61-6.

2. Bouchard JP, Barbeau A, Bouchard R, Bouchard RW. Autosomal recessive spastic ataxia of Charlevoix-Saguenay. Can J Neurol Sci 1978;5:61-9.

3. Baets J, Deconinck T, Smets K, et al. Mutations in SACS cause atypical and late-onset forms of ARSACS. Neurology 2010;75:1181-8.

4. Parkinson MH, Bartmann AP, Clayton LMS, et al. Optical coherence tomography in autosomal recessive spastic ataxia of Charlevoix-Saguenay. Brain 2018;141:989-99.

5. Harding AE. Friedreich's ataxia: a clinical and genetic study of 90 families with an analysis of early diagnostic criteria and intrafamilial clustering of clinical features. Brain 1981;104:589-620. 